\title{
NUCLEAR QUADRUPOLE RESONANCE BY FOURIER TRANSFORM OF THE FREE INDUCTION DECAY*
}

\author{
R. LENK and E. A. C. LuCKen \\ Department of Physical Chemistry, University of Geneva, 1211-Geneva 4 \\ Switzerland
}

\begin{abstract}
Fourier Transform spectrometry has been applied to nuclear quadrupole resonance (NQR). This increases the sensitivity and avoids the creation of side-bands produced by the usual superregenerative spectrometer. It provides also the possibility of determining the true spectral line-width and studying the fine structure of NQR spectral lines.
\end{abstract}

\section{INTRODUCTION}

The equivalence, under certain conditions, of the Fourier Transform of the free induction decay (FTFID) of nuclear magnetization and the corresponding slow-passage line-shape in nuclear magnetic resonance is now well-established ${ }^{1,2}$ and forms the basis of commercial high-resolution NMR spectrometers. In this context the main advantage resides in the improvement in sensitivity brought about by the FTFID technique. With certain modifications the theory of free induction decay of quadrupole resonance closely follows that of nuclear magnetic resonance ${ }^{3,4}$ and the same relationship obtains between the Fourier Transform of decay and the line-shape. In the context of NQR the gain in signal/noise ratio that this technique could bring about is perhaps of secondary importance. since it is by no means easy to search for an unknown resonance with a pulse spectrometer. In certain circumstances, for example, Zeeman studies of single crystals or powders where the operating frequency remain fixed, this aspect would, however, become significant. More important is the fact that the most commonly used and most sensitive spectrometer used for the detection of NQR is a superregenerative oscillator which considerably distorts the line-shape as well as introducing side-bands separated from the central line by integral multiples of the quench frequency. Even regenerative or crossed-coil spectrometers suffer from modulation broadening which obscures fine structure in the line-shape. Such fine structure can be expected to arise from direct or indirect spin-spin coupling or from isotope effects and have hitherto only been observed in the rare cases where such splittings exceed several $\mathrm{kHz}$.

* Research supported by the Swiss National Fund, Grant No. $258771 \mathrm{z}$. 


\section{THEORY}

The main difference between the free induction decay of a pure quadrupole system present in a polycrystalline sample and that of a similar spin $\frac{1}{2}$ system in nuclear magnetic resonance lies in the fact that the length of the pulse, $t_{\mathrm{w}}$, producing a maximum amplitude of the free induction decay is no longer given by the expression $H_{1} t_{\mathrm{w}}=\pi / 2$, but by an analogous expression, $x H_{1} t_{\mathrm{w}}=\pi / 2$, where the factor $x$ depends on the nuclear spin. For example, for $I=\frac{3}{2}, \eta=0, x$ has the value 1.774. The value of $x$ is different for a single crystal from that for a powder and also depends on the value of $\eta^{1}$. Apart from this factor, which only affects the optimum instrumental conditions. the Fourier Transform relationship between the line-shape and the free induction decay is identical with that obtaining in $\mathrm{NMR}^{5}$ and the exactitude of the relationship is subject to the same conditions in the two cases. The most important of these is $t_{\mathrm{w}} \ll 1 / 4 \Lambda$, where $\Delta$ is the width of the spectrum. The majority of pure quadrupole resonance lines have widths of not more than $10 \mathrm{kHz}-$ which implies $t_{\mathrm{w}} \ll 20 \mu \mathrm{s}-$ but in some cases much greater widths do occur and very high r.f. fields become necessary if $t_{\mathrm{w}}$ is to be kept to a satisfactory level.

The Fourier Transform procedure is of course normally applied to an accumulation of free induction decays and an important factor is the maximum pulse repetition rate. This is essentially governed by the spin-lattice relaxation time, $T_{1}$, and $T_{\mathrm{s}}$, the interval between successive scans, should be approximately $T_{\mathrm{s}} \doteq 5 T_{1}$. With the exception of ${ }^{14} \mathrm{~N}$ compounds, quadrupole spin lattice relaxation times at temperatures down to $77 \mathrm{~K}$ are seldom greater than $1 \mathrm{~s}$ and usually very much less, and quite high repetition rates are possible. Should the length of $T_{1}$ become a problem, this difficulty can be overcome by reducing $t_{\mathrm{w}}$ below the optimum ${ }^{2}$.

Another factor influencing the repetition rate is the resolving power. It can be shown ${ }^{6}$ that the relationship between the smallest frequency which can be resolved, $v_{\min }$, and the pulse interval is $v_{\min }=0.6 / T_{\mathrm{s}}$. Pure quadrupole resonance lines are usually of the order of $1 \mathrm{kHz}$ in width, but it is conceivable that it might be desirable to resolve fine structure of the order of $100 \mathrm{~Hz}$. Even this, however, implies a pulse interval of $100 \mathrm{~ms}$, which will usually be much less than that imposed by considerations of $T_{1}$.

There are thus so far two major differences between NQR and NMR in this context, both brought about by the larger line-widths and shorter relaxation times of the quadrupolar systems. The advantages in a higher allowable repetition rate that these allow are of course off-set by the higher radiofrequency powers which are necessary in NQR if the pulse-width, $t_{\mathrm{w}}$, is not to be excessively long.

If the above conditions are all satisfied, the remaining problem is to carry out the Fourier Transform operation on the accumulated free induction decay, $f(t)$ :

$$
\begin{aligned}
F(\omega) & =\int_{-\infty}^{+\infty} f(t) \exp (-\mathrm{i} \omega t) \mathrm{d} t \\
& =\int_{-\infty}^{+\infty} f(t) \cos \omega t \mathrm{~d} t-\mathrm{i} \int_{-\infty}^{+\infty} f(t) \sin \omega t=F_{\mathrm{c}}(\omega)-\mathrm{i} F_{\mathrm{s}}(\omega)
\end{aligned}
$$


The solution to the problem of performing the Fourier Transform on minicomputers is now well established and the Cooley-Tukey Fast Fourier Transform algorithm ${ }^{7}$ produces the separate cosine and sine transformscorresponding to the absorption and dispersion spectra-respectively, within a minute or so. It should be noted, however, that whereas the integration in equation (1) goes from $\infty$ to $-\infty$, we are operating in real time and can only go forwards. As it stands, therefore, one would only obtain one half of the absorption or dispersion curve if the operation mode was as above. The solution is to artificially shift the frequency origin by setting the operating frequency, $v^{\prime}$, slightly higher or lower than the resonance frequency, $v_{0}$. The free induction decay is then modulated by the difference frequency, $\omega / 2 \pi=\left(v^{\prime}-v_{0}\right)$ :

$$
f(t)=g(t) \cos 2 \pi\left(v^{\prime}-v_{0}\right)
$$

The Fourier Transform of this is then centred about $\left(v^{\prime}-v_{0}\right)$ and both halves of the spectra can be obtained provided $\left(v^{\prime}-v_{0}\right) \gg 3 \Delta v$. This, however, results in a folding of the spectrum (Figure 1 ), since it is immaterial whether $v^{\prime}$ be higher or lower than $v_{0}$ (see Appendix). Any doubt as to this can of course be eliminated by repeating the measurement at a slightly different frequency.

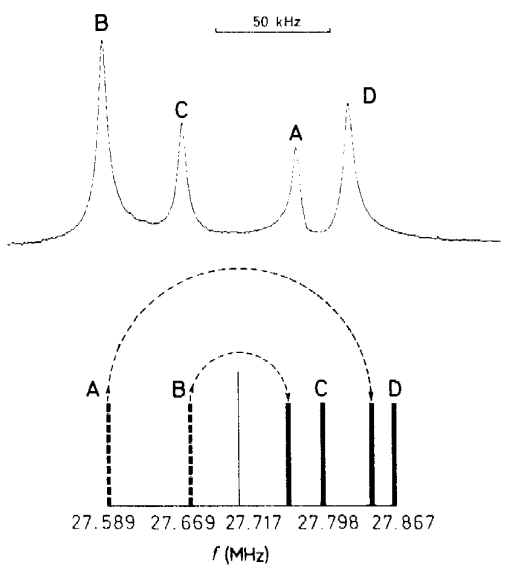

Figure 1. Folding of the spectra in ${ }^{35} \mathrm{Cl} \mathrm{NQR}$ spectrum of $\left(\mathrm{PNCl}_{2}\right)_{3}$ (room temperatures).

The question of frequency offset introduces an instrumental problem in NQR, whereas in high-resolution NMR the whole frequency range, even for polyelectronic nuclei, usually extends over only $10-20 \mathrm{kHz}$, it is quite common in NQR to have a spectrum spread over several $\mathrm{MHz}$. There are two factors which prevent the simultaneous presentation of such a spectrum. The first is that of course all the frequencies in the spectral range must be amplified and detected by the spectrometer receiver, which normally has only a restricted band-pass. This is commonly a few hundred $\mathrm{kHz}$. The second 


\section{R. LENK AND E. A. C. LUCKEN}

arises from the finite number of data points which accommodate each free induction decay. If $\Delta t$ is the dwell-time per channel, the total frequency domain is $1 / 2 \Delta t \mathrm{~Hz} z^{8}$. Thus for a dwell-time of $1 \mu \mathrm{s}-$-the lowest we have used in this study - the total frequency domain is $500 \mathrm{kHz}$. Both these considerations therefore show that the spectrum can only be displayed in intervals of a few hundred $\mathrm{kHz}$ at a time. The total spectrum must therefore be reconstituted section by section, but of course in most cases the line positions will have been obtained by a conventional spectrometer and the Fourier Transform method applied just in the region of the known resonances.

A further problem with the Fourier Transform method arises from the phase shift which is introduced by a variety of instrumental factors. There are two main sources of this. The first arises from delays introduced by the various filters in and after the phase-sensitive detector and is essentially constant at least over the $500 \mathrm{kHz}$ or so of a spectrum. The second arises mainly from the amplitude of the pulse, $H_{1}$, being insufficiently great. This phase shift is frequency-dependent over the displayed spectrum. The consequence of these phase shifts is of course that the real and imaginary parts of the Fourier Transform are now mixtures of absorption and dispersion signals.

Two methods may be forseen in the FFT programme for overcoming these phase shifts. In the first the phase shifts- - one constant and the other linearly frequency-dependent-can be applied by the operator after inspection of the transformed spectrum, and the process is repeated until pure absorption and dispersion signals are obtained. This procedure is very appropriate for NMR, where the operating frequency is constant and once the phase-shifts have been determined they remain constant and may be automatically applied thereafter. For NQR, however, they will vary from nucleus to nucleus, from frequency to frequency and from compound to compound. The correction process must thus be applied manually each time. The second method is to compute the modulus (power spectrum), i.e.

$$
F_{\mathrm{m}}(\omega)=\left\{F_{\mathrm{c}}^{2}(\omega)+F_{\mathrm{s}}^{2}(\omega)\right\}^{\frac{1}{2}}
$$

which is independent of phase shifts (see Appendix). This method has the disadvantage of broadening the line, as is shown in Figure 2 for a Lorentzian line-shape. but has the advantage of rapidity for routine measurements. Surprisingly enough, although the lines are broadened, the resolution of two overlapping lines is if anything improved, since the calculation of the modulus increases the separation between the two maxima of the composite line. This is illustrated in Figure 2 for a theoretically constructed pair of Lorentzian lines with gradually increasing separation and in Figure 3 for an experimental wide-line NMR spectrum where both absorption and power spectra are obtained.

The Lorentzian spectral line in the modulus representation $F_{\mathrm{m}}(\omega)$ is modified in the following manner :

$$
F_{\mathrm{m}}^{2}(\omega)=T_{2}^{2} /\left(1+\omega^{2} T_{2}^{2}\right)^{2}+\omega^{2} T_{2}^{4} /\left(1+\omega^{2} T_{2}^{2}\right)^{2}=T_{2}^{2} /\left(1+\omega^{2} T_{2}^{2}\right)
$$

We can see that the Lorentzian spectral line in the modulus representation is proportional to the square root of the absorption, $F_{\mathrm{c}}(\omega)$. 
(a)

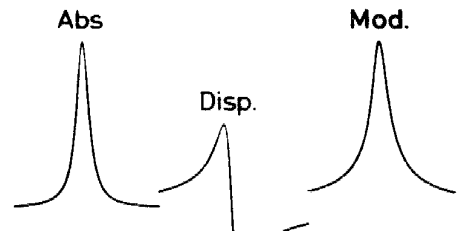

(b)

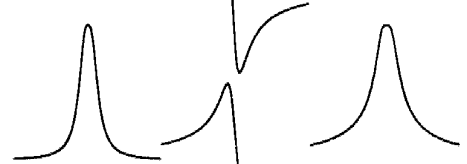

(c)

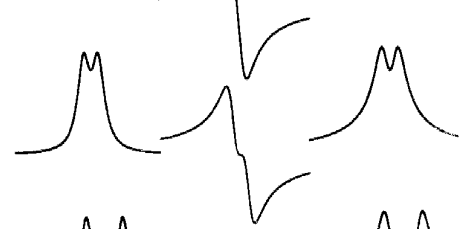

(d)

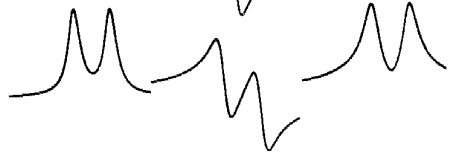

Figure 2. Computer-simulated two-line Lorentzian spectrum in the absorption, dispersion and modulus $\left(M^{2}=A^{2}+D^{2}\right)$ representations. The splitting $A$ of the spectrum is variable and the line width $2 / T_{2}$ is constant: (a) $\Lambda=0$; (b) $\Lambda=1 / T_{2}$; (c) $\Lambda=2 / T_{2}$; (d) $\Lambda=5 / T_{2}$

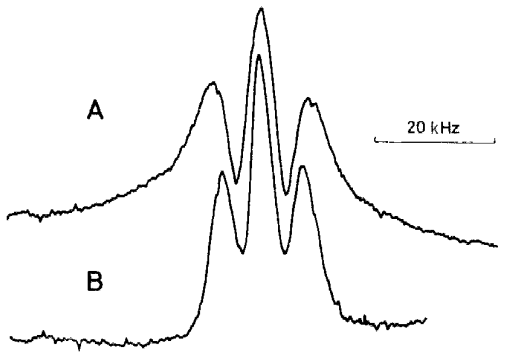

Figure 3. Comparison of an FT-NMR spectrum of MBBA liquid crystal (nematic phase) in the modulus (A) and absorption (B) representations

\section{EXPERIMENTAL}

The pulse spectrometer, forming the basis of the present equipment, is the Bruker 323-s spectrometer, operating from 4 to $60 \mathrm{MHz}$. The only modification has been to the probe unit, which was designed for insertion in a magnet pole gap and in which the sample could not be cooled below $100 \mathrm{~K}$. The probe used here (Figure 4) was based on the bridge circuit employed by Jeffrey and Armstrong. ${ }^{9}$. The maximum r.f. field-atom disposition was sufficient to produce a ' $90^{\circ}$ pulse' for ${ }^{35} \mathrm{Cl}$ nuclei in a sample volume of $1.5 \mathrm{~cm}^{3}$ in a time of $10 \mu \mathrm{s}$ at a frequency in the neighbourhood of $30 \mathrm{MHz}$. 


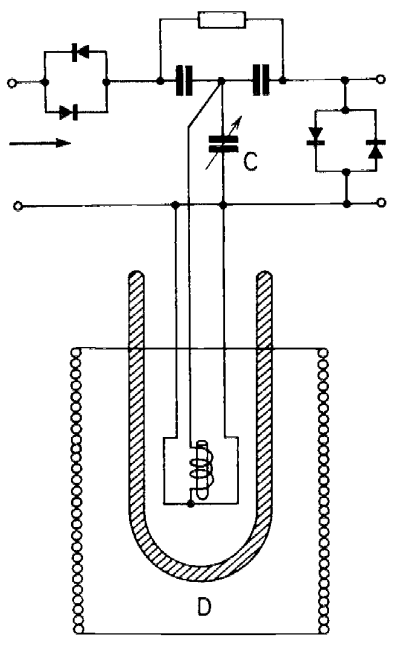

Figure 4. Probe used for FT-NQR measurements at low temperatures

Temperature control is very important in pulsed NQR spectrometry, owing to the temperature dependence of the resonance frequency. Typical temperature coefficients of ${ }^{35} \mathrm{Cl}$ resonances are $1-5 \mathrm{kHz} \mathrm{deg}^{-1}$, and since a line of $1 \mathrm{kHz}$ width will usually be accumulated for $5 \mathrm{~min}$, the temperature should not vary by more than about $0.1{ }^{\circ} \mathrm{C}$ over this period. Most measurements reported here have been carried out at $77 \mathrm{~K}$, with the sample assembly plunged directly into liquid nitrogen. At room temperature satisfactory temperature stability may be achieved by placing the coil assembly in an empty Dewar vessel and allowing the system to come to equilibrium with the pulse input, a by no means unimportant source of heat.

The output from the receiver is monitored by an oscilloscope and sampled. digitalized and averaged by a Nicolet $1074 / 4 \mathrm{~K}$ signal averager, equipped with a high-speed digitizer (SD 77) and sweep control unit (SW 77). The minimum dwell-time per channel is $1 \mu \mathrm{s}$. This combination is not entirely satisfactory for three reasons: in the first place, the minimum dwell-time is still a little long for many purposes; in the second, the SD 77 digitizer has only 4-bit conversion; and finally, the SD 77 uses half the $4 \mathrm{~K}$ memory of the signal averager as a buffer store and only averages over $2 \mathrm{~K}$ points. The Nicolet 1074 is interfaced with a PDP 8/L 4K computer and the Fourier Transform is calculated by use of the Cooley-Tukey algorithm ${ }^{7}$. In the 'Computer Control' mode the $4 \mathrm{~K}$ memory of the Nicolet 1074 is available to the PDP $8 / \mathrm{L}$, so that the combined system has an effective $8 \mathrm{~K}$ capacity. After transformation the spectrum is returned to the store of the Nicolet 1074 , where it may be viewed on an oscilloscope or read out on a chart recorder.

Of particular utility is the 'Numerical Display' accessory of the Nicolet 1074, which presents digitally the contents of any selected channel. This makes location of peak maxima, measurements of line-widths, etc., particularly easy and free from operator errors. 


\section{RESULTS}

The spectra presented here have all been measured at $77 \mathrm{~K}$. unless otherwise stated, and the figures show the power spectra.

Although, as indicated previously, increased sensitivity is not the primary aim of Fourier Transform NQR spectrometry, it is of interest to ensure that this aspect is, at least qualitatively, achieved. Figure $5(a)$ shows a ${ }^{35} \mathrm{Cl}$ spectrum measured on a Decca superregenerative spectrometer with a total scan time of 5 min and a time constant of $3 \mathrm{~s}$. while Figure $5(\mathrm{~b})$ shows the spectrum of the identical sample accumulated for $5 \mathrm{~min}$. We have made

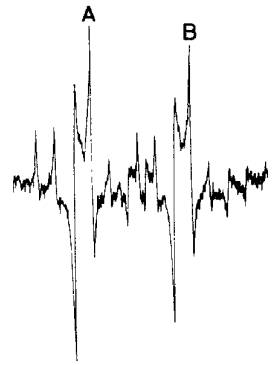

(a)

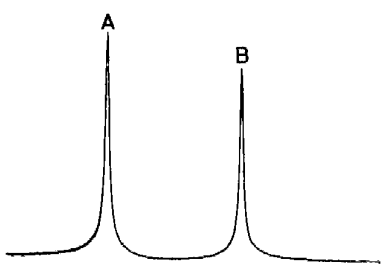

(b)

Figure 5. The ${ }^{35} \mathrm{Cl} \mathrm{NQR}$ spectra of 2,2,6,6-tetrachloro-tricyclo( $\left(5,10,0^{6,7}\right)$ octane at $35.892 \mathrm{MHz}$ (room temperatures). (a) cw-spectrum, (b) FT spectrum

no attempt to investigate the sensitivity enhancement quantitatively and systematically, but qualitatively there is no doubt as to the much improved signal/noise ratio. It should be stressed, however, that the sweep rate on the superregenerative spectrometer corresponds to approximately $4 \mathrm{MHz} / \mathrm{h}$, whereas, although a similar sweep rate could no doubt be achieved on the pulse spectrometer, it would necessitate the continuous presence of an operator. It should, however, be possible to eliminate his presence, although the effort required to do so would be by no means trivial. Figure 6 shows

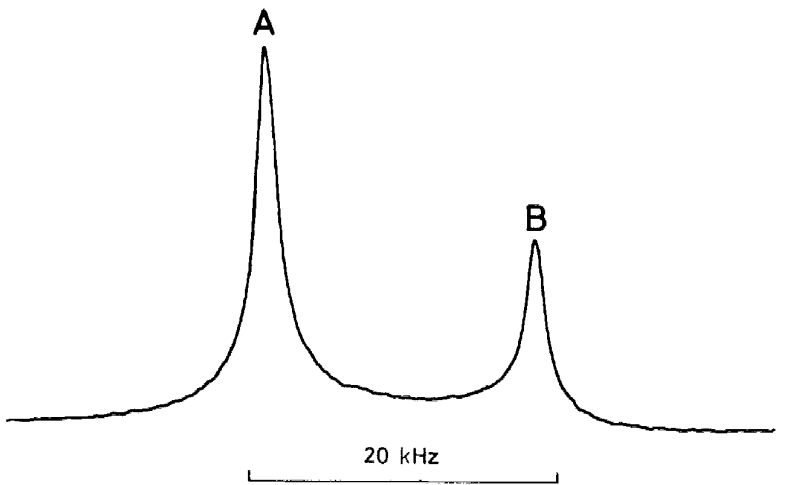

F igure $6 .{ }^{35} \mathrm{Cl} \mathrm{FT-NQR} \mathrm{spectrum} \mathrm{of} \mathrm{the} \mathrm{cyanurylchloride} \mathrm{(room} \mathrm{temperatures).} \mathrm{at} 36.290 \mathrm{MHz}$ 


\section{R. LENK AND E. A. C. LUCKEN}

another example, this time of a spectrum with lines which, according to the crystal structure, should be in a $2: 1$ intensity ratio. An example of a fourline spectrum- of $\left(\mathrm{PNCl}_{2}\right)_{3}$ - is shown of course in Figure 1, where it was used to illustrate the 'folding' phenomenon. It also illustrates a problem concerning intensity measurement. Because of the band-pass characteristics of the receiver and the power spectrum of the exciting pulse, resonances further and further removed from the exciting frequency are progressively attenuated. The crystal structure of $\left(\mathrm{PNCl}_{2}\right)_{3}$, combined with single-crystal Zeeman studies, show that lines $\mathrm{A}$ and $\mathrm{D}$ arise from pairs of equivalent chlorine atoms, whereas $\mathrm{B}$ and $\mathrm{C}$ arise from single chlorine atoms. The integrated intensities of $\mathrm{A}$ and $\mathrm{D}$ should thus be twice as great as those of $\mathrm{B}$ and $\mathrm{C}$, but this is clearly not the case. Accurate relative intensities of line close enough to be observed in the same measurements can, however, be obtained by the following device. The spectrum of two lines, frequency $v_{\mathrm{A}}$ and $v_{\mathrm{B}}$. is observed at an exciting frequency $\frac{1}{2}\left(v_{\mathrm{A}}+v_{\mathrm{B}}\right)+\delta$. where $\delta$ is a fraction of the frequency difference, $v_{\mathrm{A}}-v_{\mathrm{B}}$. The integrated intensities are measured as $I_{\mathrm{A}}^{\prime}$ and $I_{\mathrm{B}}^{\prime}$. The spectrum is again measured at $\frac{1}{2}\left(v_{\mathrm{A}}+v_{\mathrm{B}}\right)-\delta$ to yield intensities $I_{\mathrm{A}}^{\prime \prime}, I_{\mathrm{B}}^{\prime \prime}$. Provided that the band-pass function of the receiver is symmetric, the true relative intensities are given by $I_{\mathrm{A}} / I_{\mathrm{B}}$ :

$$
I_{\mathrm{A}} / I_{\mathrm{B}}=\left(I_{\mathrm{A}}^{\prime} / I_{\mathrm{B}}^{\prime} \cdot I_{\mathrm{A}}^{\prime \prime} / I_{\mathrm{B}}^{\prime \prime}\right)^{\frac{1}{2}}
$$

The following spectra are devoted to illustrating the question of fine structure in NQR lines. One of the earliest observed examples of this, the ${ }^{35} \mathrm{Cl}$ resonance in $\mathrm{BCl}_{3}$, shows two lines of unequal intensity separated by $4.15 \mathrm{kHz}$. It is now fairly certain that this is due to an isotope effect, the molecules ${ }^{10} \mathrm{BCl}_{3}(18.8 \%)$ and ${ }^{11} \mathrm{BCl}_{3}(81.2 \%)$ having slightly different frequencies, although the possibility that it arose from spin-spin coupling was also considered. Figure 7 shows the $\mathrm{FT}{ }^{35} \mathrm{Cl}$ spectrum of boron trichloride. The main doublet is clearly marked, but more interesting is the much narrower

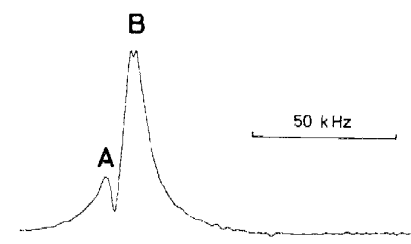

Figure 7. ${ }^{35} \mathrm{Cl}$ FT-NQR spectrum of $\mathrm{BCl}_{3}$ at $21.535 \mathrm{MHz}$

and just-resolved doublet structure of the more intense peak. It would seem very likely that this structure arises from direct or indirect $\mathrm{B}^{35} \mathrm{Cl}$ spin-spin coupling. The fact that it is not visible on the less intense peakwhich we suppose to be due to ${ }^{10} \mathrm{BCl}_{3}$ is a natural consequence of the relative magnetic moments and spins of ${ }^{11} \mathrm{~B}$ and ${ }^{10} \mathrm{~B}\left({ }^{11} \mathrm{~B}: I=3 / 2, \mu=2.6880\right.$ nuclear magnetons; ${ }^{10} \mathrm{~B}: l=3, \mu=1.8005$ nuclear magnetons). 


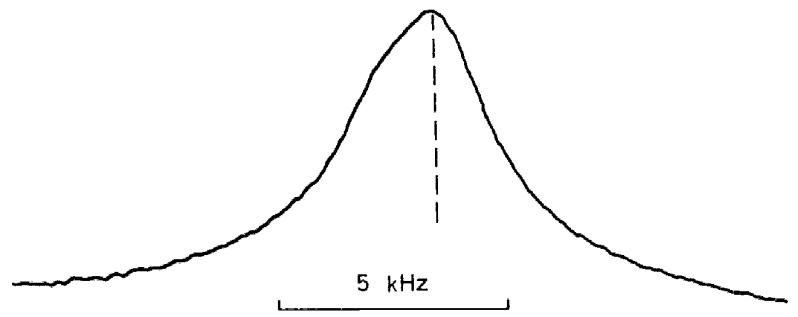

Figure 8. Presence of a shoulder in an FT-NQR spectral line of $\left(\mathrm{PNCl}_{2}\right)_{4}$ at $28.585 \mathrm{MHz}$.

Figure 8 shows one of the lines of $\left(\mathrm{PNCl}_{2}\right)_{4}$ showing a very pronounced asymmetry. A more marked shoulder is seen in one of three lines of $\mathrm{Au}_{2} \mathrm{Cl}_{6}{ }^{10}$. In both these cases the source of the underlying structure could be either isotope effects or spin-spin coupling.

The final example of fine structure is shown in Figure 9, which illustrates the Zeeman effect in the NQR spectrum of $p$-dichlorobenzene. The small external magnetic field perpendicular to the sample coil axis removes the degeneracy of the NQR transition and the original one spectral line is split in to four components.

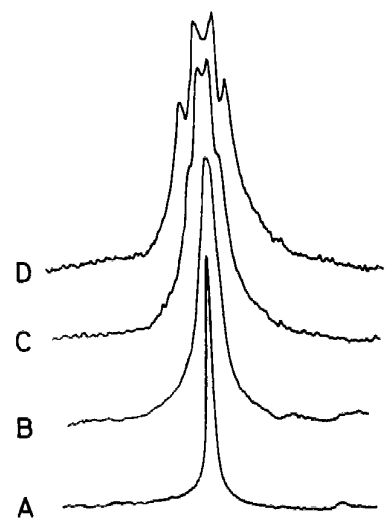

Figure 9. Line shape of the ${ }^{35} \mathrm{Cl}$ resonance of a polycrystalline paradichlorobenzene in the presence of a magnetic field parallel to the coil axis: (A) no external magnetic field; (B-D) gradually increasing field

\section{CONCLUSIONS}

In conclusion, the application of the Fourier Transform to the NQR free induction decay has produced the expected gain in sensitivity and reveals some details of fine structure, not observable by other means. 


\section{APPENDIX: GENERAL EXPRESSION FOR THE MODULUS MODE}

The free induction decay for $n$ spectral lines is given by the superposition of $n$ individual interferograms:

$$
f(t)=\sum_{i=1}^{n} g_{i}(t) \cos \left(2 \pi \gamma_{i} t+\phi_{i}\right)
$$

where $g_{i}(t)=T_{2}^{*}$ decay of the $i$ th spectral line; $\gamma_{i}=v_{\mathrm{hf}}-v_{i}\left(v_{i}\right.$ is the resonance frequency of the $i$ th spectral line); and $\phi_{i}=$ frequency-dependent phase shift of the $i$ th interferogram.

The real part of the Fourier Transform of $f(t)$ is given by:

$F_{\mathrm{c}}(v)=\sum_{i=1}^{n} \int_{0}^{\infty} g(t) \cos \left(2 \pi \gamma_{i} t+\phi_{i}\right) \cos 2 \pi v t \mathrm{~d} t$

$=\frac{1}{2} \sum_{i=1}^{n}\left[\cos \phi_{i}\left\{A_{i}\left(v+\gamma_{i}\right)+A_{i}\left(v-\gamma_{i}\right)\right\}-\sin \phi_{i}\left\{D_{i}\left(v+\gamma_{i}\right)-D_{i}\left(v-\gamma_{i}\right)\right\}\right]$

where

$$
\begin{array}{ll}
A(v)=\int_{0}^{\infty} g(t) \cos 2 \pi v t \mathrm{~d} t & \text { (absorption mode) } \\
D(v)=\int_{0}^{\infty} g(t) \sin 2 \pi v t \mathrm{dt} \quad \text { (dispersion mode) }
\end{array}
$$

Equation (A2) shows two important properties of $F_{\mathbf{R}}(v)$. (1) The phase shift $\phi_{i}$ causes the mixing of absorption and dispersion modes. (2) Any absorption or dispersion line consists of two equivalent components, shifted from the $v_{\mathrm{hf}}$ frequency by $\pm \gamma_{i}$. This is the 'mirror phenomenon' as stated in the main text and presented in Figure 1.

The similar expression may be developed for the imaginary part of the Fourier Transform, $F_{I}(v)$.

For the further development of the expression for the modulus mode, $F_{M}(v)$, we neglect the interference between the 'mirror' components and develop the functions of $(v-\gamma)$ only. The final expression is :

$$
\begin{aligned}
& F_{M}^{2}(v)=\frac{1}{4} \sum_{i=1}^{n}\left[A_{i}^{2}(-)+D_{i}^{2}(-)\right] \\
& +\frac{1}{2} \sum_{i=1}^{n} \sum_{j=2}^{n}\left[A_{i}(-) A_{j}(-) \cos \phi_{i} \cos \phi_{j}+D_{i}(-) D_{j}(-) \sin \phi_{i} \sin \phi_{j}\right. \\
& -A_{i}(-) D_{j}(-) \cos \phi_{i} \sin \phi_{j}-D_{i}(-) A_{j}(-) \sin \phi_{i} \cos \phi_{j} \\
& +D_{i}(-) D_{j}(-) \cos \phi_{i} \cos \phi_{j}+A_{i}(-) A_{j}(-) \sin \phi_{i} \sin \phi_{j} \\
& \left.\quad+D_{i}(-) A_{j}(-) \cos \phi_{i} \sin \phi_{j}+A_{i}(-) D_{j}(-) \sin \phi_{i} \cos \phi_{j}\right]
\end{aligned}
$$

where $A_{i}(-)$ is the simplified notation for $A_{i}\left(v-\gamma_{i}\right)$.

The analysis of equation (A3) shows that the modulus mode eliminates the absorption and dispersion modes mixing in two cases: 
NUCLEAR QUADRUPOLE RESONANCE BY FOURIER TRANSFORM

(1) The interference between the individual spectral lines may sometimes be neglected (particularly in NQR); in this case all cross-terms in equation (A3) vanish and $F_{M}^{2}(v)$ is given thus:

$$
F_{\mathrm{M}}^{2}(v)=\frac{1}{4} \sum_{i=1}^{n}\left[A_{i}^{2}(-)+D_{i}^{2}(-)\right]
$$

(2) The spectral lines are near to one another and the phase angles may be considered to be frequency-independent $\left(\phi_{i}=\phi\right)$ :

$$
\begin{aligned}
F_{\mathrm{M}}^{2}(v)=\frac{1}{4} \sum_{i=1}^{n}\left[A_{i}^{2}(-)+\right. & \left.D_{i}^{2}(-)\right] \\
& +\frac{1}{2} \sum_{i=1}^{n} \sum_{\substack{j=2 \\
i<j}}^{n}\left[A_{i}(-) A_{j}(-)+D_{i}(-) D_{j}(-)\right]
\end{aligned}
$$

In this case the phase angles $\phi_{i}$ and the absorption-dispersion cross-terms are eliminated, but the absorption-absorption and dispersion-dispersion cross-terms remain. The latter are for the most part responsible for 'increase of resolution', as has been shown in Figures 2 and 3.

\section{REFERENCES}

1 I. J. Loewe and R. F. Norberg, Phys. Rev. 107, 46 (1957).

R. R. Ernst and W. A. Anderson, Rev. Sci. Instrum. 37, 93 (1966).

M. Bloom, E. L. Hahn and B. Herzog, Phys. Rev. 97, 1699 (1955).

4 T. P. Das and A. K. Saha, Phys. Rev. 98, 516 (1955).

5 A. Abragam. The Principles of Nuclear Magnetism. p 214. Oxford University Press; London (1961).

${ }^{6}$ R. R. Ernst. The Application of Computer Techniques to Chemical Research, p. 61. Institute of Petroleum; London (1972).

7 J. W. Cooley and J. W. Tukey, Math. Computation, 19, 267 (1965).

8 T. C. Ferrar and E. D. Becker. Pulse \& Fourier Transform NMR, p 69. Academic Press; New York (1971).

9 K. R. Jeffrey and R. L. Armstrong, Rev. Sci. Instrum., 38, 634 (1967).

10 R. Lenk and E. A. C. Lucken, Chem. Phys. Letters, 21, 552 (1973). 